Disclaimer: This is a version of an unedited manuscript that has been accepted for publication. As a service to authors and researchers we are providing this version of the accepted manuscript (AM). Copyediting, typesetting, and review of the resulting proof will be undertaken on this manuscript before final publication of the Version of Record (VoR). During production and prepress, errors may be discovered which could affect the content, and all legal disclaimers that apply to the journal relate to this version also.

To cite this article: Ambrose A. Adegbege William P. Heath (2015): Directionality Compensation for Linear Multivariable Anti-windup Synthesis., International Journal of Control, DOI: $10.1080 / 00207179.2015 .1045556$. To link to this article:

http://dx.doi.org/10.1080/00207179.2015.1045556

This article may be used for research, teaching, and private study purposes. Any substantial or systematic reproduction, redistribution, reselling, loan, sub-licensing, systematic supply, or distribution in any form to anyone is expressly forbidden. Terms Conditions of access and use can be found at http://www.tandfonline.com/page/terms-and-conditions 


\title{
Directionality Compensation for Linear Multivariable Anti-windup Synthesis.
}

\author{
Ambrose A. Adegbege* and William P. Heath**
}

\begin{abstract}
We develop new synthesis procedures for optimizing anti-windup control applicable to open-loop exponentially stable multivariable plants subject to hard bounds on the inputs. The optimizing antiwindup control falls into a class of compensator commonly termed directionality compensation. The computation of the control involves the on-line solution of a low-order quadratic program in place of simple saturation. We exploit the structure of the quadratic program to incorporate directionality information into the off-line anti-windup synthesis using a decoupled architecture similar to that proposed in the literature for anti-windup schemes with simple saturation. We demonstrate the effectiveness of the design compared to several schemes using a simulated example.
\end{abstract}

\section{INTRODUCTION}

Most practical control problems must deal with constraints imposed by equipment limitations such as actuator nonlinearities. One approach that has received much attention in dealing with such problems is the anti-windup technique [2], [3], [4], [5], [6], [7], [8]. It is also a common practice to incorporate an additional artificial non-linearity (directionality compensator) in multivariable anti-windup designs to address the problem of directionality [9], [10], [11], [12], [13], [14]. In general, the design of a directionality compensator is carried out independently of the linear control design and with the assumption that the resulting optimizing structure inherits the

* Department of Electrical and Computer Engineering, The College of New Jersey, PO Box 7718, Ewing, NJ 08628. Email: adegbega@tenj.edu

** Control Systems Centre, School of Electrical and Electronics Engineering, The University of Manchester, Sackville Street Building, M13 9PL, UK. Email: william.heath@manchester.ac.uk

Preliminary results of this work have been published in the proceedings of the IEEE conference on decision and control, Orlando, $2011[1]$. 
stability of the unsaturated loop (e.g. [10], [12]). Such directionality compensators often take the form of solutions to some convex optimization problems; these are solved either implicitly ([15]) or explicitly ([10], [11], [12]) on-line during control computation. When the control policy is obtained by an explicit solution of an on-line optimization problem at each time step, the resulting scheme is termed optimizing (e.g. [16], [17]). In this paper, we distinguish such on-line optimization from the off-line synthesis required before implementation. We assume the control action is available instantaneously, as the underlying optimization is low-order, independent of state, piecewise affine in the solution space and can be solved order of magnitude faster than the plant bandwidth [18].

The main contribution of this paper is the synthesis of anti-windup with both stability and performance guarantees for systems incorporating directionality compensation in the form of a quadratic program (QP). In particular, we note that the information from the directional compensator that is resolved on-line can be incorporated into the off-line anti-windup synthesis to guarantee closed-loop stability as well as improved performance.

This paper extends the preliminary results of [1], where development was restricted to the internal model control (IMC) structure of [9], [15] and it is a natural generalization of [4] to cases where the control non-linearity is coupled and satisfy a generalized sector condition. The resulting off-line synthesis is characterized by two matrices: $F$ from the linear anti-windup conditioning and $E$ from the directionality compensation. We design $E$ to minimize some integral squared error performance objective and then synthesize $F$ via a convex search over linear matrix inequalities (LMIs).

Other related works include [17], [19], [20] where sufficient conditions for closed-loop stability were derived in terms of the Karoush-Kuhn-Tucker (KKT) conditions associated with the input non-linearity. These approaches allow only for a posteriori stability checks when the anti-windup compensator has already been designed using an existing design technique (e.g. [15]). Others are [21], [22] where non-diagonal stability multipliers are employed in the analysis of systems with decoupled and repeated nonlinearities. Synthesis using the non-diagonal multipliers of ([21], [22]) may be problematic [23, Remark 3]. In [2], an algebraic loop was deliberately introduced into the static anti-windup configuration for improved performance. Under certain conditions ([24]), the algebraic loop leads to a QP whose solution may be considered a directionality compensator. However, the well-posedness and practical implementations of such algebraic loops are non- 
trivial [25]. The synthesizing LMI of [2] is also not always feasible (see e.g.[3]) and the choice of performance specification do not relate directly to the true goal of anti-windup design which is the swift recovery of linear performance ([4], [26]). Here, we exploit an extra design freedom for incorporating the system's directional characteristics as well as stability and performance requirements for a dynamic anti-windup synthesis which is always feasible. This extra design freedom has previously been exploited in [27] but only to eliminate algebraic loop in the antiwindup construction.

Notations: Let $\mathcal{L}_{2}^{m}$ be the Hilbert space of $\mathbb{R}^{m}$ valued functions $f$ on $[0, \infty)$ such that $\int_{0}^{\infty}|f(t)|^{2} d t$ is finite. The expression $\langle f, g\rangle$ stands for the inner product of signals $f, g \in \mathcal{L}_{2}^{m}$ defined by $\int_{0}^{\infty} f(t)^{T} g(t) d t$. For a general operator $\Pi: \mathcal{L}_{2}^{m} \rightarrow \mathcal{L}_{2}^{m}, \Pi^{*}$ denotes the adjoint. Let $\Pi$ be a bounded self-adjoint operator satisfying $\Pi=\Pi^{*}$; then a bounded operator $\psi: \mathcal{L}_{2}^{m} \rightarrow \mathcal{L}_{2}^{m}$ is said to satisfy the Integral Quadratic Constraint (IQC) defined by $\Pi$ or simply $\psi \in \operatorname{IQC}(\Pi)$ if the following inequality holds [28]

$$
\left\langle\left[\begin{array}{l}
u \\
v
\end{array}\right], \Pi\left[\begin{array}{l}
u \\
v
\end{array}\right]\right\rangle \geq 0 \text { for all } v=\psi(u), u \in \mathcal{L}_{2}^{m} .
$$

The signs $\preceq$ and $\succeq$ denote element by element inequalities.

\section{Problem SetuP}

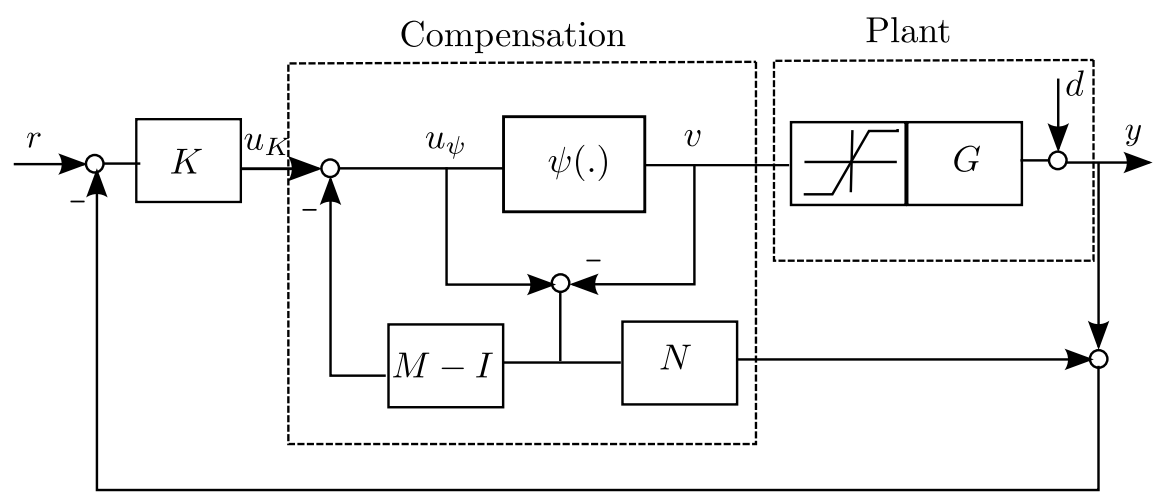

Fig. 1: Configuration for anti-windup and directionality compensation.

We consider the optimizing structure of Fig. 1 . The plant is given by $y=G u+d$ with only bounded $u$ admissible and where $G$ represents the plant dynamics which are assumed to be perfectly known. To avoid confusion, we discriminate between $u_{K}, u_{l i n}$ and $u_{\psi}$ as follows: $u_{K}$ 
is the output of controller $K$ for the compensated closed-loop, $u_{l i n}$ is the output of controller $K$ when the closed-loop is in the linear range i.e. the input constraints are not active and $u_{\psi}$ is the input to the directionality compensator defined by $\psi($.$) . We denote the state space realizations$ of $G$ and its right co-prime factorization $G=N M^{-1}$ respectively as

$$
G \sim\left[\begin{array}{c|c}
A & B \\
\hline C & D
\end{array}\right] \text { and }\left[\begin{array}{c}
M \\
N
\end{array}\right] \sim\left[\begin{array}{c|c}
A+B F & B \\
\hline F & I \\
C+D F & D
\end{array}\right]
$$

where $F$ is such that $A+B F$ is Hurwitz. The linear controller denoted as $K$ is assumed to have been designed to meet some acceptable linear stability and performance criteria. This implies that the unconstrained closed-loop system

$$
\begin{aligned}
& y_{\text {lin }}=(I+G K)^{-1} G K r+(I+G K)^{-1} d \\
& u_{\text {lin }}=K(I+G K)^{-1}(r-d)
\end{aligned}
$$

is internally stable [29]. Usually, the linear controller $K$ is designed such that the closed loop system (3) is decoupled. The signal $y_{\text {lin }}$ is the unconstrained (linear) plant output. The exogenous signals $r$ and $d$ represent the reference and the disturbance signals respectively. The control input bound is modeled using a saturation function block as follows:

$$
\hat{u}=\operatorname{sat}(u)=\left[\begin{array}{c}
\operatorname{sat}\left(u_{1}\right) \\
\vdots \\
\operatorname{sat}\left(u_{m}\right)
\end{array}\right] \text { where } \operatorname{sat}\left(u_{i}\right)= \begin{cases}u_{i}^{\max } & u_{i} \succ u_{i}^{\text {max }} \\
u_{i} & u_{i}^{\text {min }} \preceq u_{i} \preceq u_{i}^{\text {max }} \\
u_{i}^{\text {min }} & u_{i} \prec u_{i}^{\text {min }}\end{cases}
$$

denotes the saturation non-linearity associated with each of the manipulated input $u_{i}(t)$ for some $u_{i}^{\min } \preceq 0$ and $u_{i}^{\max } \succeq 0$.

Since the multivariable saturation non-linearity (4) acts element by element, the direction of the actual plant input vector $\hat{u}$ necessarily differs from that of the controller output vector $u$ [9], [13]. Such directional change may be unduly amplified especially for plants with strong structural couplings [9]. For such class of systems, a naive application of the classical antiwindup techniques has been shown to result in significant performance deterioration or even instability [30], [9]. This performance loss is generally attributed to the effects of directionality (e.g. [31], [10], [11], [12]). The artificial non-linearity, represented as $\psi($.$) , is introduced in Fig. 1$ such that the difference between the unconstrained response $y_{l i n}$ and the constrained response $y$ 
is minimized in some sense. Let $E \in \mathbb{R}^{m \times m}$ be a non-singular structural matrix that represents the plant's directional characteristics; the directional compensator is such that its optimal solution $v$ minimizes the Euclidean norm of the difference between $E v$ and $E u_{\psi}$ while not violating the constraints imposed by the actuator limits. This constrained weighted least distance problem can be stated as

$$
\min _{v} \frac{1}{2}\left(v-u_{\psi}\right)^{T} H\left(v-u_{\psi}\right), \text { subject to } u_{i}^{\min } \preceq v_{i} \preceq u_{i}^{\max }, i=1, \cdots, m
$$

or in the following standard form

$$
\psi\left(u_{\psi}\right)=\arg \min _{v} \frac{1}{2} v^{T} H v-v^{T} H u_{\psi}, \text { subject to } L v \preceq b \text { with } b \succeq 0
$$

where $H=E^{T} E=H^{T}>0 \in \mathbb{R}^{m \times m}$. The fixed terms $L \in \mathbb{R}^{2 m \times m}$ and $b \in \mathbb{R}^{2 m}$ in the inequality constraints are obtained from (4) as

$$
L=\left[\begin{array}{ll}
-I_{m}, & I_{m}
\end{array}\right]^{T} \text { and } b=\left[-\left(u^{\min }\right)^{T}, \quad\left(u^{\max }\right)^{T}\right]^{T}
$$

where $u^{\min }=\left[u_{1}^{\min }, \cdots, u_{m}^{\min }\right]^{T} \preceq 0$ and $u^{\max }=\left[u_{1}^{\max }, \cdots, u_{m}^{\max }\right]^{T} \succeq 0$ are the lower and upper bounds on the control inputs respectively. It is important to note that $v=\operatorname{sat}\left(u_{\psi}\right)$ solves (6) when $E$ is diagonal or the identity and that $v$ is admissible for any choice of $E$ (i.e. $v=\operatorname{sat}(v))$. Since $v$ is always admissible, we can safely ignore the saturation block preceding the plant model $G$ in Fig. 1. The objective function of (6) is strictly convex by virtue of $H$ being positive definite. Since the constraints describe a box which contains the origin, the feasible set is not empty [17], the quadratic program always has a unique solution [32].

\section{EQUIVALENT REPRESENTATIONS}

We take advantage of the structure of the non-linearity $\psi($.$) to obtain equivalent representations$ of the optimizing anti-windup framework of Fig. 1. These alternative representations are directly linked to the two design variables $F$ and $E$, and hence provide insights on their design or synthesis. First, we transform $\psi($.$) into related nonlinearities satisfying some sector conditions.$

Lemma 1: Let $v=\psi\left(u_{\psi}\right)$ be the quadratic program defined by (6) and let $w=\xi\left(u_{\psi}\right)$ be the quadratic program

$$
\xi\left(u_{\psi}\right)=\arg \min _{w} \frac{1}{2} w^{T} H w, \text { subject to } L u_{\psi}-L w \preceq b,
$$


with $H=E^{T} E=H^{T}>0$ and where $L$ and $b$ are defined as in (7). The interconnection of $w=\xi\left(u_{\psi}\right)$ with $v=u_{\psi}-w$ is equivalent to $v=\psi\left(u_{\psi}\right)$.

Lemma 2: Let the quadratic program (6) be set as $v=\psi\left(u_{\psi}\right)$ with $H=E^{T} E=H^{T}>0$ and $L$ and $b$ are defined as in (7). Let $\bar{v}=\phi(\bar{u})$ be the quadratic program

$$
\phi(\bar{u})=\arg \min _{\bar{v}} \frac{1}{2} \bar{v}^{T} \bar{v}-\bar{v}^{T} \bar{u}, \text { subject to } R \bar{v} \preceq b .
$$

Suppose $\phi(\bar{u})=E \psi\left(u_{\psi}\right), \bar{u}=E u_{\psi}$ and $R=L E^{-1}$, then by input-output scaling, $\psi(u)$ and $\phi(\bar{u})$ are equivalent. Furthermore, $\phi(\bar{u})$ belongs to the sector[0 1$]$.

Remark 1: If $E$ is chosen to be diagonal or the identity, $\psi($.$) and \xi($.$) reduce to the decentral-$ ized saturation and dead-zone non-linearities respectively (see e.g. [19], [20]). This is the case in most existing anti-windup formulations where $E$ is used to eliminate algebraic loops (e.g. [27], [4]).

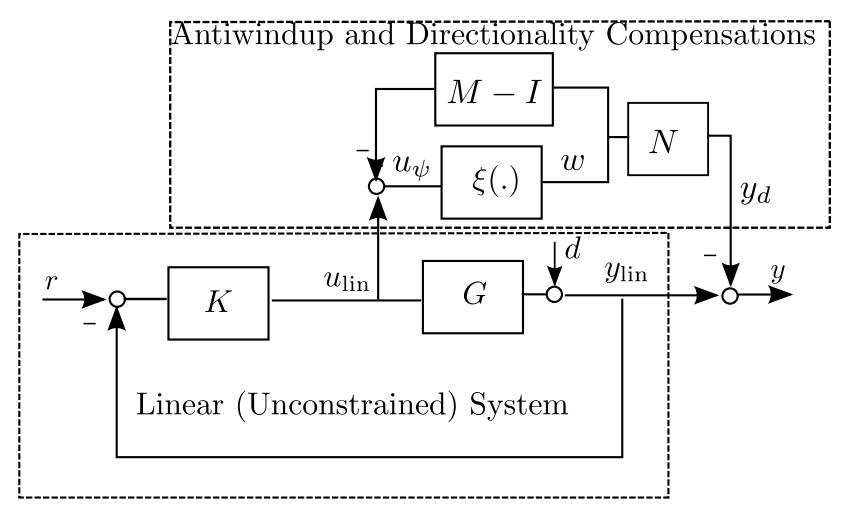

Fig. 2: Equivalent Structure I.

Using the transformation in Lemma 1 and the relationships in (3), the optimizing structure in Fig. 1 can be represented in the decoupled structure of Fig. 2. This decoupled structure is attractive for anti-windup synthesis (e.g. [4], [33]) and allows for easy incorporation of information from the directionality compensator $\psi($.$) or \xi($.$) into the offline synthesis. Alternatively, using$ the linear transformations in Lemma 2, the optimizing anti-windup structure in Fig. 1 can be re-arranged as shown in Fig. 3 where $\widetilde{M}$ and $\widetilde{N}$ are given by:

$$
\left[\begin{array}{c}
\widetilde{M} \\
\widetilde{N}
\end{array}\right] \sim\left[\begin{array}{c|c}
A+B F & B E^{-1} \\
\hline F & E^{-1} \\
C+D F & D E^{-1}
\end{array}\right] .
$$




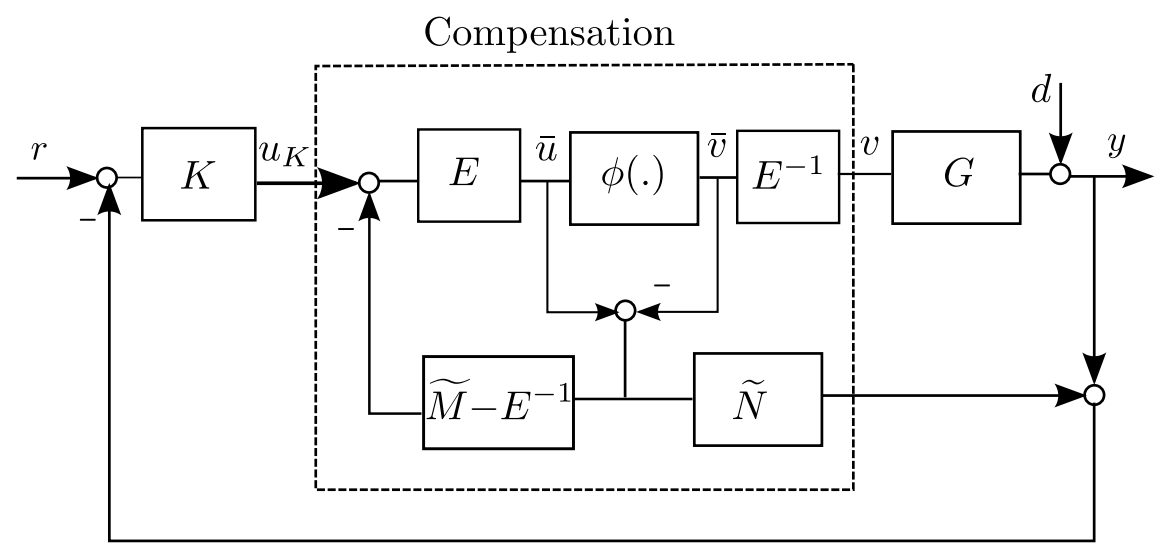

Fig. 3: Equivalent Structure II.

with $F$ and $E$ being design variables and $E$ is invertible. The co-prime factorization (10) is more general than (2) as it offers an extra degree of freedom via the static matrix $E$ which can be exploited in the anti-windup design. The anti-windup design then reduces to finding appropriate parameters $F$ and $E$ which ensure both the stability and the performance of the closed-loop system. This interpretation is similar to the co-prime-factor parametrization in [27], [33], but here the optimizing framework provides some additional insights as to the role and the choice of $E$. We also advocate that $E$ should not be used as a free design parameter but should be chosen based on some structural characteristics of the plant. From the equivalent structure of Fig. 3, the block $E^{-1}$ may be considered a static pre-compensator for the plant and may be chosen such that the response is decoupled either in the transient period or at steady states (e.g. [12], [17], [34]). Similarly, the block $E$ may be considered a static post-compensator for the controller and may be chosen such that $K E$ is decoupled (e.g. [10]). Existing directionality compensation schemes [10], [12], [17] correspond to particular choices of $E$. We highlight some specific choices here and refer readers to [35] for detailed derivations.

1. Optimization based Conditioning Technique [10]

$$
E=\lim _{s \rightarrow \infty}[K(s)]^{-1}
$$

2. Optimal Directionality Compensation [12]

$$
E=\lim _{s \rightarrow \infty}\left[\operatorname{diag}\left(s^{r_{i}}\right) G(s)\right]
$$


where $r_{i}=\min \left(r_{i 1}, r_{i 2}, \ldots, r_{i m}\right)$ and $r_{i, j}$ is the relative degree of output $y_{i}$ with respect to manipulated input $u_{j}[12]$. In this case, it is usual to call $E$ the characteristic or decoupling matrix of $G(s)$ as it captures the structural couplings between its input and output channels [34].

3. Steady State Directionality Compensation [17], [34]

$$
E=\lim _{s \rightarrow 0}[G(s)]
$$

Thus, it is possible to choose $E$ such that during saturation, the map between the admissible input $v$ and the plant output $y$ is decoupled at certain frequencies. Suppose that $E$ is chosen based on the above structural considerations such that performance deterioration due to the effects of directionality is minimized in some sense. Then, $F$ can be chosen based on convex search such that the anti-windup system is guaranteed stable and that recovery of linear performance is hastened. For the special case where $E$ is diagonal, the directionality compensation (6) reduces to the repeated saturation non-linearity (4) (see Remark 1). In this instance, $v=\operatorname{sat}\left(u_{\psi}\right)$ is the optimal solution to (6) and the inclusion of $E$ in the anti-windup synthesis is of no use (see [27] and also Corollary 2). We discuss in the next section a two-step procedure for the synthesis of $F$ and $E$ and also comment on the procedure for the simultaneous synthesis of $F$ and $E$.

\section{ANTI-WINDUP COMPENSATOR SYNTHESIS}

We use the machinery of integral quadratic constraints (IQC) to synthesize appropriate antiwindup compensator while incorporating information from the directionality compensator. We only consider static IQCs, since it is our intention to employ such IQCs in the convex synthesis of a suitable anti-windup compensator. Consider the feedback structure of Fig. 4. Standard IQC

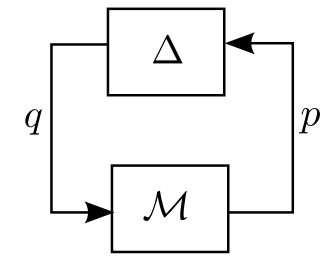

Fig. 4: Interconnections for Synthesis

theory [28] gives sufficient conditions for the stability of such interconnection if for $\Delta \in \operatorname{IQC}(\Pi)$, 
the following inequality is satisfied

$$
\left[\begin{array}{c}
\mathcal{M}(j \omega) \\
I
\end{array}\right]^{T} \Pi\left[\begin{array}{c}
\mathcal{M}(j \omega) \\
I
\end{array}\right]<0 \quad \forall \omega \in \mathbb{R} .
$$

Suppose we define $y_{d}=y-y_{\text {lin }}$, a fictitious block $\Delta_{p}$ satisfying the $\mathcal{L}_{2}$ gain condition $\left\|u_{\text {lin }}\right\|^{2}-\frac{1}{\gamma^{2}}\left\|y_{d}\right\|^{2} \leq 0$ with $\gamma>0$ (e.g. [36]) and the nonlinear map $w=\xi\left(u_{\psi}\right)$ satisfying $w^{T} H\left(w-u_{\psi}\right) \leq 0$ with $w=u_{\psi}-v$ and $H=H^{T}>0$. The closed-loop structure Fig. 2 can be transformed into the feedback interconnection of Fig. 4 with $\Delta=\operatorname{diag}\left(\xi, \Delta_{p}\right)$ and

$$
\mathcal{M}=\left[\begin{array}{cc}
I-M & I \\
-N & 0
\end{array}\right]
$$

where $p=\left[\begin{array}{l}u_{\psi} \\ y_{d}\end{array}\right]$ and $q=\left[\begin{array}{c}w \\ u_{l i n}\end{array}\right]$. The performance requirement that the $\mathcal{L}_{2}$ gain of the map from $u_{\text {lin }}$ to $y_{d}$ be less than $\gamma>0$ is equivalent to checking the interconnection of Fig. 4 is stable for all $\Delta_{p}$ which is norm bounded by $1 / \gamma$. This is a well-known convention (see [37, section 4.3] and [21, comment after Theorem 2]). Also, such a performance specification has been observed to be central to the anti-windup design problem (e.g. [4], [38]) for the saturated case. Using appropriate IQCs satisfied by the input-output maps of $\xi$ and $\Delta_{p}$, we state the following result.

Theorem 1: Given a stable linear time invariant plant $G$ with co-prime factorization (2). Let $\mathcal{M}$ be given by (15) and $\Delta=\operatorname{diag}\left(\xi, \Delta_{p}\right)$ be a bounded operator such that $\xi$ and $\Delta_{p}$ satisfy respectively the IQCs conditions

$$
\begin{aligned}
& \left\langle\left[\begin{array}{l}
u_{\psi} \\
w
\end{array}\right],\left[\begin{array}{cc}
0 & H \Lambda \\
\Lambda H & -(H \Lambda+\Lambda H)
\end{array}\right]\left[\begin{array}{c}
u_{\psi} \\
w
\end{array}\right]\right\rangle \geq 0 \forall w=\xi\left(u_{\psi}\right), u_{\psi} \in \mathcal{L}_{2}^{m} \text { and } \\
& \left\langle\left[\begin{array}{c}
y_{d} \\
u_{l i n}
\end{array}\right],\left[\begin{array}{cc}
I & 0 \\
0 & -\gamma^{2} I
\end{array}\right]\left[\begin{array}{c}
y_{d} \\
u_{l i n}
\end{array}\right]\right\rangle \geq 0 \forall u_{l i n}=\Delta_{p}\left(y_{d}\right), y_{d} \in \mathcal{L}_{2}^{m} .
\end{aligned}
$$

Suppose there exist $P=P^{T}>0$, diagonal $\Lambda>0$ and $\alpha=\gamma^{2}>0$ satisfying matrix inequality (18)

$$
\left[\begin{array}{ccc}
\tilde{A}^{T} P+P \tilde{A}^{T} & P B-F^{T} H \Lambda & 0 \\
B^{T} P-\Lambda H F & -\Lambda H-H \Lambda & \Lambda H \\
0 & H \Lambda & -\alpha I
\end{array}\right]+\left[\begin{array}{c}
\tilde{C}^{T} \\
D^{T} \\
0
\end{array}\right]\left[\begin{array}{c}
\tilde{C}^{T} \\
D^{T} \\
0
\end{array}\right]^{T}<0
$$


with $\tilde{A}=A+B F, \tilde{C}=C+D F$. Then, the feedback interconnection of $\mathcal{M}$ and $\Delta$ is stable for all $u_{\psi}, y_{d} \in \mathcal{L}_{2}^{m}$ and the $\mathcal{L}_{2}$-gain from $u_{\text {lin }}$ to $y_{d}$ is less than $\gamma$.

Theorem 1 is not suitable for synthesis but allows for an a posteriori stability check when both the anti-windup and the directionality compensations have already been designed (i.e $F$ and $E$ are both fixed). The IQC condition (16) is a generalization of the multivariable circle criterion for sector-bounded nonlinearities [39]. In what follows, we modify (18) for convex synthesis. We first consider the two-step design procedure.

\section{A. Two-step design procedure for $F$ and $E$}

The following corollary provides an anti-windup synthesis procedure for a given directionality compensation parameter $E$.

Corollary 1: Consider the optimizing structure of Fig. 2 with a stable linear time invariant plant $G$ described by (2) and non-linearity described by the quadratic program (8). Suppose $E$ is fixed with $H=E^{T} E$ and $T=H^{-1}$. Further Suppose there exist $Q=Q^{T}>0, \gamma>0$, diagonal $U>0$ and $X$ satisfying the linear matrix inequality (19)

$$
\left[\begin{array}{cccc}
A Q+Q A^{T}+B X+X^{T} B^{T} & B U T-X^{T} & 0 & Q C^{T}+X^{T} D^{T} \\
T U B^{T}-X & -U T-T U & I & T U D^{T} \\
0 & I & -\gamma I & 0 \\
C Q+D X & D U T & 0 & -\gamma I
\end{array}\right]<0
$$

Then, there exists a plant-order anti-windup compensator which renders the interconnection of Fig. 2 stable with an $\mathcal{L}_{2}$ gain from $u_{\text {lin }}$ to $y_{d}$ less than $\gamma$. The design parameter $F$ characterizing the co-prime factorization (2) is recovered using $F=X Q^{-1}$ where $X$ and $Q$ are feasible solutions of LMI (19).

After $E$ has been chosen based on some structural considerations as highlighted in section III, the design parameter $F$ can be synthesized using (19) which is linear in its variables.

\section{B. Simultaneous Synthesis of $F$ and $E$}

Corollary 2: Consider the optimizing structure of Fig. 2 with a stable linear time invariant plant $G$ described by (2) and non-linearity described by the quadratic program (8). Suppose 
there exist $Q=Q^{T}>0, \gamma>0, W$ and $X$ satisfying the linear matrix inequality (20)

$$
\left[\begin{array}{cccc}
A Q+Q A^{T}+B X+X^{T} B^{T} & B W-X^{T} & 0 & Q C^{T}+X^{T} D^{T} \\
W B^{T}-X & -W-W^{T} & I & W D^{T} \\
0 & I & -\gamma I & 0 \\
C Q+D X & D W & 0 & -\gamma I
\end{array}\right]<0
$$

Then, there exists a plant-order anti-windup compensator which renders the interconnection of Fig. 2 stable with an $\mathcal{L}_{2}$ gain from $u_{\text {lin }}$ to $y_{d}$ less than $\gamma$. The design parameters $F$ and $E$ characterizing the co-prime factorization (10) are recovered using $F=X Q^{-1}, E=\sqrt{W^{-1} U}$ where $X, Q$ and $W$ are feasible solutions of LMI (20) and $U>0$ is arbitrary.

Note that the feasibility of LMI (20) guarantees the invertibility of $W$. From the $(2,2)$ element of LMI (20), we have that $-W-W^{T}<0$. It follows that $W>0$ and hence $W^{-1}$ exists.

Remark 2: Note that matrix $W$ in LMI (20) is not restricted to be diagonal as in other LMIbased anti-windup designs (e.g. [4, LMI (13)]). Although allowing $W$ to assume a full block structure may be considered a greater degree of freedom in the choice of $F$, the recovered $F$ from LMI (20) tends to place the poles of the anti-windup compensator far into the left halfplane. This feature is characteristic of LMI-based dynamic anti-windup schemes (see [4], [40] for details).

Remark 3: The main difference between Corollaries 1 and 2 is the explicit inclusion of $E$ via $T=H^{-1}$ in (19). LMI (20) is independent of $H$. This may suggest that $H$ can be chosen arbitrarily via $H=W^{-1} U$ where $U$ is an arbitrary matrix. It is important to note that a naive choice of $U$ may not preserve the structure of the directionality compensator. An alternative is to restrict $W$ in LMI (20) to be symmetric (i.e $W=W^{T}$ ) while $U$ is chosen to be diagonal positive definite. With this, any $H$ recovered from $H=W^{-1} U$ (for $W=W^{T}$ satisfying (20)) is guaranteed to be symmetric positive definite (i.e. $H=H^{T}>0$ ). It follows that any such $H$ will preserve the structure of the directionality compensator. Similar conditions have earlier been employed to guarantee the well-posedness of algebraic loops (e.g. [41], [25]).

In existing anti-windup literature (e.g. [2], [3], [4]), $U$ is usually chosen such that $H=I$. In this case, LMI (19) reduces to a special case of (20) where $W$ is diagonal (compare with [4, LMI (13)]). However, when $H$ is chosen as discussed in section III, LMI (19) allows for the incorporation of the plant directional characteristics into the anti-windup optimization. 
Remark 4: Since the $\mathcal{L}_{2}$ performance channels in Corollary (1) are scaled versions of those in Corollary (2), the attained $\mathcal{L}_{2}$ gains are essentially the same (e.g. see [36, pg 95]). $\square$

Remark 5: Note that there is always a choice of $F$ such that LMIs (19) and (20) are feasible. Choosing $F=0$ implies $M=I$. In this instance, LMI (19) reduces to a version of the bounded real lemma (e.g. [36]) and the $\mathcal{L}_{2}$ gain computed when $H=I$ corresponds to the infinity-norm of the stable plant $G$. This case also recovers the conventional IMC anti-windup structure of [9].

Since the solution of LMI (20) tends to produce fast dynamics with large closed-loop poles, it is common, for ease of implementation, to constrain the anti-windup poles to regions comparable to the unconstrained closed-loop poles. In the spirit of [42], the anti-windup poles can be constrained to a region formed by the intersection of the negative half s-plane and a disc of radius $r$. This can easily be achieved by solving in tandem with LMI (20) the following LMI region [42]

$$
\left[\begin{array}{cc}
-r P & A P+B X \\
P A^{T}+X^{T} B^{T} & -r P
\end{array}\right]<0
$$

where $r$ is the radius of the disk. Similarly, by incorporating robustness constraints [4] into LMI (20), the anti-windup poles can be restricted to favorable regions for implementation.

\section{Simulation Example}

In order to demonstrate the effectiveness of the proposed design procedure, we consider an ill-conditioned example typical of distillation column control [29]. This is a well-studied problem because of the strong directionality and interaction that exist in the plant as well as its high sensitivities to diagonal input nonlinearities and uncertainties. We compare three antiwindup design approaches, namely the optimal directionality compensation scheme [12], the dynamic anti-windup without directionality compensation [4] and the proposed anti-windup with directionality compensation (Corollary 1 ).

The plant model is given by the transfer function matrix

$$
G(s)=\frac{1}{75 s+1}\left[\begin{array}{ll}
0.878 & -0.864 \\
1.082 & -1.096
\end{array}\right]
$$


with both inputs constrained as $\left|u_{i}\right| \leq 100, i=1,2$. In the absence of control input saturations, the linear controller is designed to achieve a completely decoupled closed-loop response described by

$$
G_{F}(s)=\frac{1}{1.43 s+1} I .
$$

The unity feedback controller which achieves this decoupled response is given by [29]

$$
K(s)=\frac{75 s+1}{1.43 s}\left[\begin{array}{cc}
45.38 & -35.77 \\
44.80 & -36.23
\end{array}\right] \text {. }
$$

The plant's characteristic matrix is obtained as $\frac{1}{75}\left[\begin{array}{ll}0.878 & -0.864 \\ 1.082 & -1.096\end{array}\right]$ with condition number 141.732. For the directionality compensation scheme [12], we chose $H=E^{T} E$ with $E$ as the plant's non-singular characteristic matrix. Fig. 5 shows the input and output responses of the plant to a set-point changes from $\left[\begin{array}{ll}0 & 0\end{array}\right]^{T}$ to $\left[\begin{array}{ll}0.7 & 0\end{array}\right]^{T}$ at time $t=0$ and from $\left[\begin{array}{ll}0.7 & 0\end{array}\right]^{T}$ to $\left[\begin{array}{ll}0.7 & 0.4\end{array}\right]^{T}$ at time $t=50$ for the different compensation schemes. Note that the unconstrained case requires a very aggressive control action during transients to achieve the decoupled response. During the transient periods following the two set-point changes, control actions due to the directionality compensator never violate the saturation limits. During the first transient, $u_{1}$ stays on the positive saturation limit while $u_{2}$ gradually approaches it. This process is reversed during the second transient where $u_{2}$ stays on the negative saturation limit while $u_{1}$ approaches it. For the saturated case (without anti-windup and directionality compensations), the saturation limits are violated during the two transient periods leading to clipping of both controls at the saturation limits. The effects of clipping are clear on the output response: sluggishness and inverse response. The directionality scheme [12] results in an improved transient response as compared to the saturated (uncompensated) response. Note that this scheme does not offer any stability guarantee. While the problem of directionality is solved by incorporating directionality compensation, the proposed scheme (Corollary 1) recovers linear performance faster and it is closest to the unconstrained response. This superior performance confirms the benefit of combining the optimality of an online optimization with the efficiency of convex off-line anti-windup synthesis while guaranteeing closed-loop stability.

Finally, we compare the proposed scheme (Corollary 1) with that of [4, LMI 23]. Following [4], we consider three different performance-robustness weight combinations, namely $w_{p}=$ 

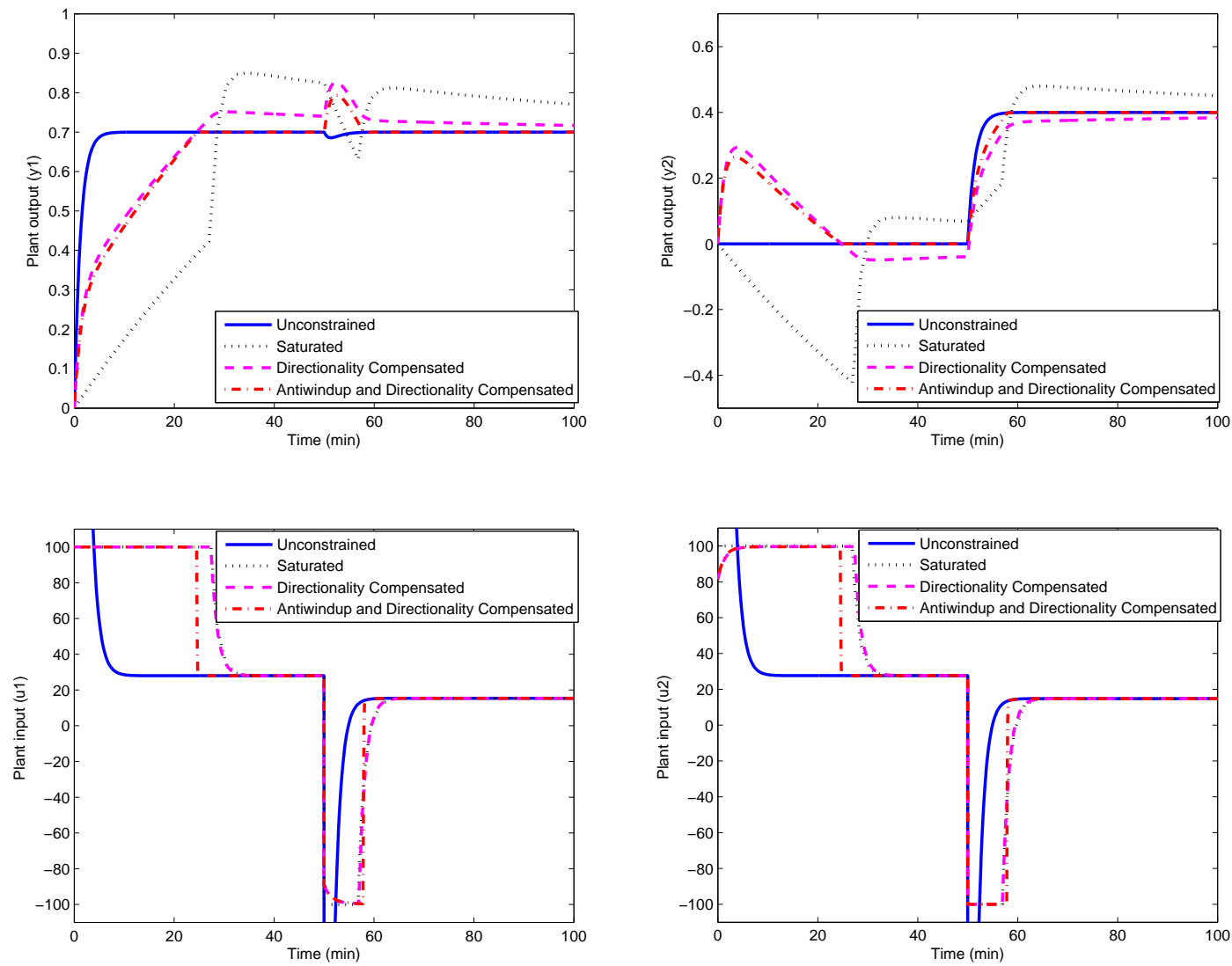

Fig. 5: Responses of the unconstrained (solid), saturated (Dotted), directionality compensated [12] (Dashed) and anti-windup with directionality compensation (Dashdotted) to a step reference. The superior performance of the anti-windup with directionality compensation over [12] can be attributed to its efficiency and swiftness in recovering linear performance after a period of nonlinear operation.

$0.01, W_{r}=1$ (case 1), $w_{p}=1, W_{r}=0.01$ (case 2) and $W_{p}=1, W_{r}=1$ (case 3). The feedback gain recovered from LMI (19) is

$$
F_{H}=\left[\begin{array}{ll}
162.1456 & -308.6913 \\
307.7003 & -454.2451
\end{array}\right] \text {. }
$$


For the three different cases of [4], we have

$$
\begin{aligned}
F_{\text {Case } 1} & =\left[\begin{array}{cc}
0.2623 & -0.2635 \\
-0.2635 & 0.2631
\end{array}\right] * 10^{-7}, F_{\text {Case } 2}=\left[\begin{array}{cc}
-20.1877 & 20.2183 \\
20.7024 & -20.7379
\end{array}\right] \\
\text { and } F_{\text {Case } 3} & =\left[\begin{array}{cc}
-0.1539 & 0.1541 \\
0.1541 & -0.1543
\end{array}\right] .
\end{aligned}
$$

As discussed in Remark 5 (also in [4]), case 1 recovers the internal model control (IMC) anti-windup since $F_{\text {Case } 1} \approx 0$. It is well-known that the IMC anti-windup is robust to inputmultiplicative uncertainties but may result in a very poor performance ([9], [4], [8]). In case 2, performance is emphasized over robustness while case 3 places equal emphasizes on robustness and performance. The input and output responses of the three different cases are compared to those of the proposed scheme in Fig. 6. Note that for all the three cases considered, the dynamic anti-windup [4] seeks to restore the plant inputs to the linear region as quickly as possible and hence the initial sluggish and inverse (on the second channel) responses observed in Fig. 6. As for the proposed scheme, only one of the control inputs is allowed to stay on the constraint during each of the transient periods. This ensured that the plant is driven in the right direction eliminating both the initial sluggish and inverse responses.

For completeness, we mention that Corollary 2 yields similar responses to [4] when the closedloop poles are subjected to same restrictions. Note that for this case we can choose $H$ as the identity (i.e. $H=I$ ). Without restrictions, the feedback gain $F$ recovered from LMI (20) is given by

$$
F_{I}=\left[\begin{array}{cc}
-1.4552 & 1.4575 \\
0.2047 & -0.2052
\end{array}\right] * 10^{5}
$$

This results in a compensator with very fast poles requiring a very high sampling frequency for implementation. These fast poles can be constrained to a suitable region either by solving LMI (21) (e.g. with $r=0.05$ ) in tandem with LMI (20) or by applying the robustness and performance weights of [4].

\section{CONCLUSiONS}

We have presented a multivariable optimizing anti-windup design which guarantees closedloop stability while compensating for the effects of both windup and directionality. Directionality 

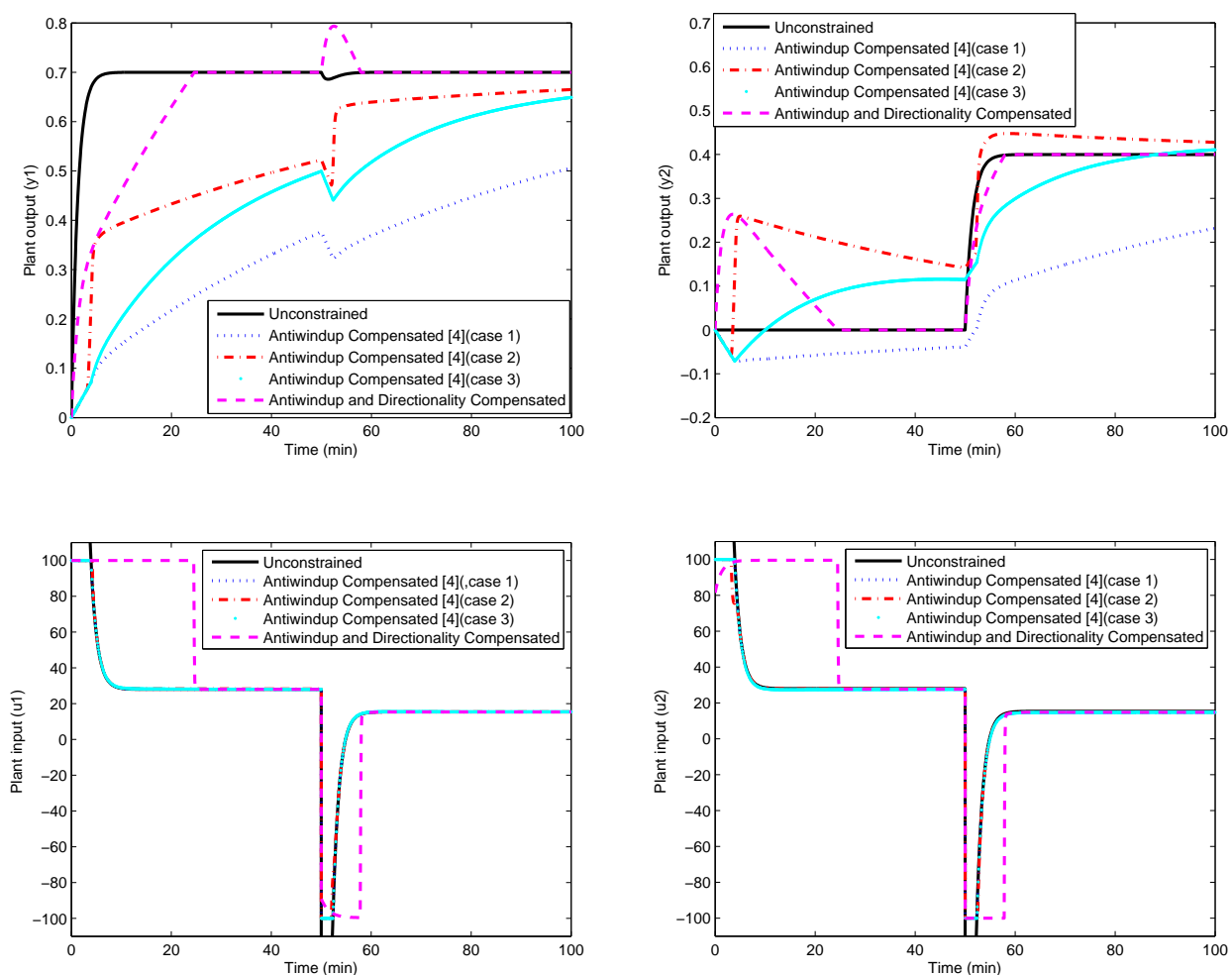

Fig. 6: Responses of anti-windup schemes with (Corollary 1) and without [4] directionality compensation: unconstrained (solid), [4, Case 1] (Dotted), [4, Case 2] (Dashdot), [4, Case 3] (Point) and LMI-based anti-windup with directionality compensation 1 (Dashed) to set-point changes from $\left[\begin{array}{ll}0 & 0\end{array}\right]^{T}$ to $\left[\begin{array}{ll}0.7 & 0\end{array}\right]^{T}$ at time $t=0$ and from $\left[\begin{array}{ll}0.7 & 0\end{array}\right]^{T}$ to $\left[\begin{array}{ll}0.7 & 0.4\end{array}\right]^{T}$ at time $t=50$. The antiwindup with directionality compensation can be seen to have a significant improvement over [4] in that it combines the optimality of directionality compensation with the efficiency of model recovery anti-windup techniques.

compensation is achieved through an on-line optimization while windup is addressed through an off-line convex dynamic anti-windup synthesis. The resulting synthesis problem is characterized by two gain matrices $F$ and $E$. In particular, we advocate that $E$ should not be used as a freedesign parameter but should be chosen based on the structural characteristics of the plant. Such structural information can then be incorporated into the convex off-line anti-windup synthesis to guarantee both closed-loop stability and performance. The simulated examples demonstrate the benefits that ensue: both from introducing directionality compensation into an anti-windup 
structure and from applying our proposed design procedures. The results are especially beneficial when the plant is ill-conditioned or has lightly damped modes. We have, however, restricted our discussions to the nominal case where there are no model uncertainties. An area of further work is to incorporate robustness into the optimizing anti-windup design (see [43]).

\section{APPENDIX}

To prove Theorem 1, we need to establish the relations between the nonlinearities $\psi$ and $\xi$. This follows from the proofs of Lemmas 1 and 2 below.

Proof of Lemma 1: The necessary and sufficient KKT conditions [32] for $\xi$ are given by

$$
H w-L^{T} \lambda=0, L u_{\psi}-L w-b+s=0, s \succeq 0, \lambda \succeq 0, \text { and } \lambda^{T} s=0 .
$$

If we substitute $w=u_{\psi}-v$ into (27), we obtain

$$
H v-H u_{\psi}+L^{T} \lambda=0, L v-b+s=0, s \succeq 0, \lambda \succeq 0, \text { and } \lambda^{T} s=0 .
$$

The conditions in (28) are exactly the KKT conditions for $\psi$. It follows that if $w$ is the unique optimal solution of (8), then the optimal solution of (6) is uniquely determined by $v=u_{\psi}-w$.

Proof of Lemma 2: The necessary and sufficient KKT conditions [32] for $\phi$ are given by

$$
\bar{v}-\bar{u}-R^{T} \lambda=0, R \bar{v}-b+s=0, s \succeq 0, \lambda \succeq 0, \text { and } \lambda^{T} s=0 .
$$

Equivalence follows by substituting $\bar{v}=E v, \bar{u}=E u_{\psi}$ and $L=R E$ into (29) and pre-multiplying the first condition by $E^{T}$ (since $E$ is invertible) to obtain (28). Pre-multiplying the first KKT condition in (29) by $\bar{v}^{T}$ and substituting gives

$$
\bar{v}^{T} \bar{v}-\bar{v}^{T} \bar{u}=-b^{T} \lambda \leq 0
$$

Hence, we may say $\phi(\bar{u})^{T}[\phi(\bar{u})-\bar{u}] \leq 0$ or analogously $\phi(\bar{u}) \in \operatorname{sector}[0, I]$

Proof of Theorem 1: From (30) and using the relation $w=u_{\psi}-v$, we have the following generalized sector condition

$$
w^{T} H w-w^{T} H u_{\psi} \leq 0 .
$$

For any diagonal $\Lambda>0$, the inequality $w^{T} \Lambda H\left(w-u_{\psi}\right) \leq 0$ also holds. In IQC notation, we can write $\xi \in \operatorname{IQC}\left(\Pi_{\xi}\right)$ with $\Pi_{\xi}=\left[\begin{array}{cc}0 & H \Lambda \\ \Lambda H & -(H \Lambda+\Lambda H)\end{array}\right]$. Hence, IQC condition 
holds for $\xi$. Also, the $\mathcal{L}_{2}$ gain performance requirement in terms of the fictitious operator $\Delta_{p}$ can be expressed in the IQC notation as $\Delta_{p} \in \operatorname{IQC}\left(\Pi_{\Delta_{p}}\right)$ where $\Pi_{\Delta_{p}}=\left[\begin{array}{cc}I & 0 \\ 0 & -\gamma^{2} I\end{array}\right]$. Then $\Delta=\operatorname{diag}\left(\phi, \Delta_{p}\right) \in \mathrm{IQC}(\Pi)$ where $\Pi$ is the diagonal augmentation of $\Pi_{\phi}$ and $\Pi_{\Delta_{p}}$ given as

$$
\Pi=\left[\begin{array}{cccc}
0 & 0 & H \Lambda & 0 \\
0 & I & 0 & 0 \\
\Lambda H & 0 & -(\Lambda H+H \Lambda) & 0 \\
0 & 0 & 0 & -\gamma^{2} I
\end{array}\right]
$$

with diagonal $\Lambda>0$. From (2), the state space realization for $\mathcal{M}$ in (15) is derived as

$$
\mathcal{M} \sim\left[\begin{array}{c}
\dot{x} \\
u_{\psi} \\
y_{d}
\end{array}\right]=\left[\begin{array}{c|cc}
A+B F & B & 0 \\
\hline-F & 0 & I \\
-(C+D F) & -D & 0
\end{array}\right]\left[\begin{array}{c}
x \\
w \\
u_{l i n}
\end{array}\right] .
$$

Using (32), (33) and the IQC frequency condition (14), the application of KYP lemma [44] leads to the matrix inequality condition

$$
\left[\begin{array}{ccc}
P \tilde{A}+\tilde{A}^{T} P+\tilde{C}^{T} \tilde{C} & P B-F^{T} H \Lambda+\tilde{C}^{T} D & 0 \\
B^{T} P+D^{T} \tilde{C}-\Lambda H F & -\Lambda H-H \Lambda+D^{T} D & \Lambda H \\
0 & H \Lambda & -\gamma^{2} I
\end{array}\right]<0
$$

where $\tilde{A}=A+B F$ and $\tilde{C}=C+D F$ and where $P=P^{T}>0$. Rearranging (34) gives (18).

Proof of Corollary 1: Substituting $X=F Q$ into (18) and followed by repeated congruence transformations using $\operatorname{diag}\left(I, T^{-1}, I, I\right)$ and $\operatorname{diag}\left(Q^{-1}, U^{-1}, I, I\right)$ gives

$$
\left[\begin{array}{cccc}
\tilde{A}^{T} Q^{-1}+Q^{-1} \tilde{A} & Q^{-1} B-F^{T} T^{-1} U^{-1} & 0 & \tilde{C}^{T} \\
B^{T} Q^{-1}-U^{-1} T^{-1} F & -U^{-1} T^{-1}-T^{-1} U^{-1} & U^{-1} T^{-1} & D^{T} \\
0 & T^{-1} U^{-1} & -\gamma I & 0 \\
\tilde{C} & D & 0 & -\gamma I
\end{array}\right]<0 .
$$

Substituting for $Q^{-1}=P, U^{-1}=\Lambda$ and $T^{-1}=H$ in (35) followed by the application of Schur's complement gives (18). The result follows by applying Theorem 1.

Proof of Corollary 2: Substituting $W=U T$ in LMI (20) gives LMI (19) and the result follows by applying Theorem 1 . 


\section{REFERENCES}

[1] A. A. Adegbege and W. P. Heath, "Anti-windup synthesis for optimizing internal model control," in The Proceedings of the 50th IEEE Conference on Decision and Control, Orlando, Florida, December 2011, pp. 5503-5508.

[2] E. F. Mulder, M. V. Kothare, and M. Morari, "Multivariable anti-windup controller synthesis using linear matrix inequalities," Automatica, vol. 37, pp. 1407-1416, 2001.

[3] G. Grimm, J. Hatfiled, A. R. Teel, M. Turner, and L. Zaccarian, "Anti-windup for stable linear systems with input saturation: an LMI based synthesis," IEEE Trans. Automatic Control, vol. 48, no. 9, pp. 1509-1525, 2003.

[4] M. C. Turner, G. Herrmann, and I. Postlethwaite, "Incorporating robustness requirements into antiwindup design," IEEE Trans. Automatic Control, vol. 52, no. 10, pp. 1842-1855, 2007.

[5] S. Tarbouriech and M. Turner, "Anti-windup design: an overview of some recent advances and open problems," IET Control Theory Appl., vol. 3, no. 1, pp. 1-19, 2009.

[6] L. Zaccarian and A. R. Teel, Modern Anti-windup Synthesis. Princeton University Press, New Jersey, 2011.

[7] G. Valmórbida, S. Tarbouriech, M. Turner, and G. Garcia, "Anti-windup design for saturating quadratic systems," Systems Control Letters, vol. 62, no. 5, pp. 367 - 376, 2013.

[8] R. Morales, G. Li, and W. P. Heath, "Anti-windup and the preservation of robustness against structured norm-bounded uncertainty," International Journal of Robust and Nonlinear Control, vol. 24, pp. 2640-2652, 2014.

[9] P. J. Campo and M. Morari, "Robust control of processes subject to saturation nonlinearities," Computers and Chemical Engineering, vol. 14, no. 4/5, pp. 343-358, 1990.

[10] Y. Peng, D. Vrančić, R. Hanus, and S. R. Weller, “Anti-windup designs for multivariable controllers," Automatica, vol. 34, no. 12 , pp. $1559-1565,1998$.

[11] C. Chen and M. Perng, "An optimization-based anti-windup design for mimo control systems," International Journal of Control, vol. 69, no. 3, pp. 393-418, 1998.

[12] M. Soroush and S. Valluri, "Optimal directionality compensation in processes with input saturation non-linearities," International Journal of Control, vol. 72, no. 17, pp. 1555-1564, 1999.

[13] D. Horla, "On directional change and anti-windup compensation in multivariable control systems," Int. J. Appl. Math. Comput. Sci., vol. 19, no. 2, pp. 281-289, 2009.

[14] A. R. King-Hans, W. P. Heath, and R. Alli-Oke, "Two-stage multivariable imc antiwindup (tmia) control of a quadruple tank process using a plc," in Control Applications (CCA), 2014 IEEE Conference on, Antibes, France, October 2014, pp. 1681-1686.

[15] A. Zheng, M. V. Kothare, and M. Morari, "Anti-windup design for internal model control," International Journal of Control, vol. 60, no. 5, pp. 1015-1024, 1994.

[16] J. A. Primbs, "The analysis of optimization based controllers," Automatica, vol. 37, pp. 933-938, 2001.

[17] W. P. Heath, A. G. Wills, and J. A. G. Akkermans, "A sufficient condition for the stability of optimizing controllers with saturating actuators," International Journal of Robust Nonlinear Control, vol. 15, pp. 515-529, 2005.

[18] Y. Wang and S. Boyd, "Fast model predictive control using online optimization," Control Systems Technology, IEEE Transactions on, vol. 18, no. 2, pp. 267-278, 2010.

[19] J. A. Primbs and M. Giannelli, "Kuhn-Tucker-based stability conditions for systems with saturation," IEEE Trans. Automatic Control, vol. 46, no. 10, pp. 1643-1647, 2001.

[20] G. Li, W. P. Heath, and B. Lennox, "Concise stability conditions for systems with static nonlinear feedback expressed by a quadratic program," IET Control Theory Appl., vol. 2, no. 7, pp. 554-563, 2008. 
[21] F. J. D’Amato, M. A. Rotea, A. Megreski, and U. T. Jönsson, "New results for analysis of systems with repeated nonlinearities," Automatica, vol. 37, no. 5, pp. 739-747, 2001.

[22] V. V. Kulkarni and M. Safonov, "All multipliers for reapeated monotone nonlinearities," IEEE Trans. Automatic Control, vol. 47, no. 7, pp. 1209-1212, 2002.

[23] C. Tarbouriech, C. Prieur, and J. Gomes da Silva Jr., "Stability and stabilization of systems presenting nested saturations," IEEE Trans. Automatic Control, vol. 51, no. 8, pp. 1364-1371, 2006.

[24] A. Syaichu-Rohman, R. H. Middleton, and M. M. Seron, "A multivariable nonlinear algebraic loop as a QP with applicaions to MPC," in Proceedings of the European Control Conference, Cambridge, September 1-4 2003.

[25] A. A. Adegbege and W. P. Heath, "Multivariable algebraic loops with complementarity constraints enforcing some KKT conditions," in 52nd Annual Allerton Conference on Communication, Control, and Computing (Allerton), 2014, Monticello, Illinois, September 2014, pp. 1033-1039.

[26] A. R. Teel and N. Kapoor, "The $\mathcal{L}_{2}$ anti-windup probelm: Its definition and solution," in The Proceedings of the 4th European Control Conference, Brussels, Belgium, July 1997.

[27] M. C. Turner, I. Postlethwaite, and G. Herrmann, "Further results on full-order antiwindup synthesis: Exploiting the stability multiplier," in 6th IFAC Nonlinear Control System Design Symposium (NOLCOS), Stuttgart, 2004.

[28] A. Megretski and A. Rantzer, "System analysis via integral quadratic constraints," IEEE Trans. Automatic Control, vol. 42, no. 6, pp. 819-830, 1997.

[29] S. Skogestad and I. Postlethwaite, Multivariable feedback control-analysis and design. West Sussex: John Willey and Sons, Ltd., 2005.

[30] J. C. Doyle, R. S. Smith, and D. F. Enns, "Control of plants with input saturation nonlinearities," in Proceedings of the American Control Conference, 1987, pp. 1034-1039.

[31] K. S. Walgama and J. Sternby, "Conditioning technique for multiinput multioutput processes with input saturation," in Proceedings of the IEE Part D, vol. 140(4), 1993, pp. 231-241.

[32] R. Fletcher, Practical Methods of Optimization. Chichester: John Willey \& Sons, 1987.

[33] G. Li, G. Herrmann, D. P. Stoten, J. Tu, and M. C. Turner, “A novel robust disturbance rejection anti-windup framework," International Journal of Control, vol. 84, no. 1, pp. 123-137, 2011.

[34] R. Gessing, "Implementability of regulation and partial decoupling of mimo plants," in Control and Automation, 2006. MED '06. 14th Mediterranean Conference on, Ancona, Italy, June 2006, pp. 1-6.

[35] A. A. Adegbege and W. P. Heath, "Internal model control design for input constrained multivariable processes," AIChE, vol. 57, no. 12, pp. 3459-3472, 2011.

[36] S. Boyd, L. E. Ghaoui, E. Feron, and V. Balakrishnan, Linear Matrix Inequalities in System and Control Theory. Society for Industrial and Applied Mathematics, Philadelphia, PA, USA, 1994.

[37] M. Kothare and M. Morari, "Multivariable anti-windup controller synthesis using multi-objective optimization," in American Control Conference. Proceedings of the 1997, Albuquerque, New Mexico, Jun 1997, pp. 3093-3097.

[38] S. Crawshaw and G. Vinnicombe, "Anti-windup synthesis for guaranteed $L_{2}$ performance," in Proceedings of the 39th IEEE conference on Decision and Control, Sidney, Australia, 2000, pp. 1063-1068.

[39] W. P. Heath and G. Li, "Multipliers for model predictive control with structured input constraints," Automatica, vol. 46, no. 3, pp. 562-568, 2010.

[40] B. Hencey and A. Alleyne, “An anti-windup technique for LMI regions,” Automatica, vol. 45, pp. 2344-2349, 2009.

[41] G. Grimm, A. R. Teel, and L. Zaccarian, "Establishing the lipschitz properties of multivariable algebraic loops with 
incremental sector nonlinearities," in Proceedings of the 42nd IEEE Conference on Decision and Control, Maul Hawaii, December 2003.

[42] M. Chilali and P. Gahinet, " $H_{\infty}$ design with pole placement constraints: An LMI approach," IEEE Trans. Automatic Control, vol. 41, no. 3, pp. 358-367, 1996.

[43] A. A. Adegbege, "Constrained internal model control," PhD. Thesis, The University of Manchester, 2011.

[44] A. Rantzer, "On the Kalman-Yakubovich-Popov lemma," Systems \& Control Letters, vol. 28, pp. 7-10, 1996. 